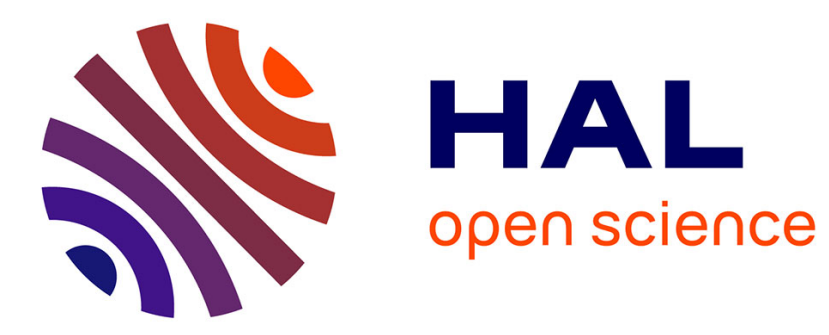

\title{
Wave Excitation Force Estimation for Wave Energy Converters of the Point-Absorber Type
}

\author{
Hoai-Nam Nguyen, Paolino Tona
}

\section{To cite this version:}

Hoai-Nam Nguyen, Paolino Tona. Wave Excitation Force Estimation for Wave Energy Converters of the Point-Absorber Type. IEEE Transactions on Control Systems Technology, 2018, 26 (6), pp.2173 - 2181. 10.1109/TCST.2017.2747508 . hal-01628474

\section{HAL Id: hal-01628474 \\ https://hal-ifp.archives-ouvertes.fr/hal-01628474}

Submitted on 3 Nov 2017

HAL is a multi-disciplinary open access archive for the deposit and dissemination of scientific research documents, whether they are published or not. The documents may come from teaching and research institutions in France or abroad, or from public or private research centers.
L'archive ouverte pluridisciplinaire HAL, est destinée au dépôt et à la diffusion de documents scientifiques de niveau recherche, publiés ou non, émanant des établissements d'enseignement et de recherche français ou étrangers, des laboratoires publics ou privés. 


\title{
Wave excitation force estimation for wave energy converters of the point absorber type
}

\author{
H.-N. Nguyen ${ }^{\dagger}$, P. Tona ${ }^{\dagger}$
}

\begin{abstract}
Advanced control strategies play a crucial role in increasing the energy extraction capacity of wave energy converters (WECs). So far, the most promising control schemes have predominantly been studied in simulation, based on the idealized assumption of the wave excitation force availability in real time. In practical WEC implementations, this is not the case, since this force cannot be measured directly when the WECs are running. Hence the force has to be estimated via measurements of other quantities. Two approaches are presented in this paper to fulfill this objective. The first approach is based on a Kalman filter coupled with a random-walk model of the wave excitation force, while a receding horizon - unknown input estimation approach is employed for the second one. The proposed estimation methods are evaluated by using real measurements from a laboratory scale WEC.
\end{abstract}

\section{INTRODUCTION}

A wave energy converter (WEC) is a device used to produce electrical energy from wave-induced motion. Several studies have shown that one of the key aspects for maximizing the energy yield of many WECs is the control of the dynamic response of the device to wave conditions, also defined as hydrodynamic control [1]. More precisely, we consider here the response control of the WEC captor, or primary converter, whose task is to transfer energy from wave to oscillating body, via the power-take off (PTO) system, responsible of a further energy conversion, generally to electricity. A schematic example of a WEC (of the point absorber type) is shown in Figure 1: a float (the captor) oscillates under the action of waves and is connected to a PTO system; the PTO, by exercising an appropriate force on the float, converts its mechanical energy into electrical energy. The PTO can be a simple linear electric generator, or a more complex multistage device, such as a hydraulic motor connected to a rotary electric generator. Hydrodynamic control of such a WEC can be performed using the PTO as an actuator and adjusting the force it exercises on the oscillating body serving as a captor, in order to maximize the extracted energy.

Different approaches to WEC hydrodynamic control have been proposed in the literature. In the second half of the 70s, to improve the energy capture of his well-known "nodding duck" [2], Salter implemented a practical control approach, where the PTO torque opposing the body oscillation was computed as the sum of three terms, respectively proportional to the displacement, the velocity and the acceleration of the pitching body. Even nowadays, this control strategy, often referred to, somewhat inaccurately, as "PID" control (because the three terms correspond to a proportional-integral-derivative

$\dagger$ Control, Signal and System Department - IFP Energies Nouvelles, Rond-point de l'echangeur de Solaize BP3, 69360 Solaize, France hoai-nam.nguyeneifpen. fr, paolino.tonalifpen.fr

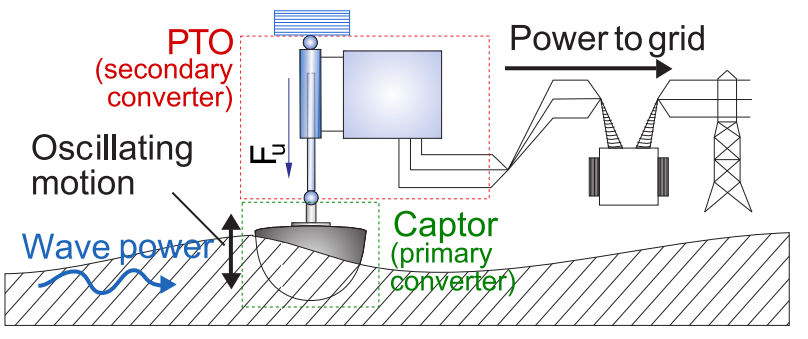

Fig. 1. Schematic diagram of a generic wave energy converter

action on velocity) is still very popular with WEC developers, because of its simplicity. As shown in [3] for the Wavestar WEC, it can yield reasonable energy capture levels, though still far below the theoretical optimum [4].

To improve wave energy conversion, several alternative control strategies have been proposed. Latching control is one of the earliest methods in this area [5], [6], [7]. The basic idea is to lock the point absorber when its velocity is zero, and wait for the most favorable moment to release it again. In this way, the velocity of the point absorber can be brought in phase with the wave excitation force, and the system is in resonance. In [8], it is shown that the performance can be improved up to $20 \%$ compared to standard PI control, by combining latching control and a short-term prediction of the wave excitation force, that is, the force exerted by the passing wave.

Another solution, that has been drawing a lot of attention in the last decade, is Model Predictive Control (MPC) [9], [10], [11]. In this strategy, a model is used to predict the future response of a system. At each sampling time interval, an MPC algorithm attempts to optimize future system behavior by computing a sequence of future control actions. The first input in the optimal sequence is then applied to the plant, and the entire calculation is repeated at subsequent sampling time intervals. The main advantage of MPC applied to WECs is that it is capable of energy conversion rates close to the theoretical optimum. In addition, state and input constraints can be considered in a natural way. However, it requires a procedure to predict the future values of the wave excitation force over the prediction horizon.

In [12], another approach is proposed to improve energy harvesting performance, the so called simple and effective realtime control. Based on the assumption of the availability of the wave excitation force, the basic idea is to calculate a reference optimal velocity. Then a control law is designed to make WEC velocity follow the reference one. By simulation, it is reported that the performance can be improved up to $25 \%$ compared 
to PI control.

It can be observed that, to achieve optimal energy conversion, all the three control strategies mentioned above rely on the assumption that the instantaneous wave excitation force is available. However, this is not the case in WEC practical implementations, since wave excitation force cannot be measured directly when the WECs are in operation. Therefore, wave excitation has to be estimated via the measurement of other quantities. In the MPC context, for instance, the complete control scheme must include a block implementing an algorithm for this purpose, as shown in Fig. 2.

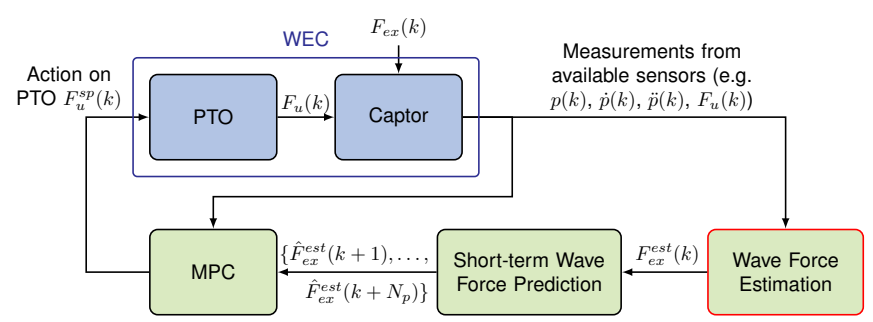

Fig. 2. Wave excitation force estimation in the context of MPC

In [13], to estimate the current value of wave excitation force, a combination of a bank of independent harmonic oscillators and a Luenberger observer is proposed. The strategy was tested on a real WEC system, but the reported experimental results show a relatively high phase lag in the estimated signal compared to the measured signal.

In [14], by considering the wave excitation force as a time-varying sinusoid, an extended Kalman filter approach is presented. However, no experimental result is reported. In addition, it is obvious that the approach may only be effective for very narrow-banded wave forces.

By combining several pressure sensors at discrete points on the buoy surface with the buoy heave position, and with an extended Kalman filter, another approach is proposed in [15], which also estimates the derivatives of wave excitation force. However, its computational complexity might be high and, in addition, extra pressure sensors are required.

Two novel solutions to estimate the wave excitation force are proposed in this paper. The first solution is based on a linear Kalman filter coupled with a random-walk model for the wave excitation force, while a receding horizon estimator is developed for the second one. All implementation aspects such as computational complexity and accuracy are investigated. The performance of the proposed solutions are compared using data, collected in the wave basin of Aalborg University, on a small-scale wave energy converter prototype, manufactured by the Wavestar company. The main features of the proposed solutions are:

1) No extra pressure sensors are required. Only measurements (or estimates) of captor position, captor velocity, and PTO force are needed.

2) The values of current wave excitation force are estimated with almost no time delay.

3) No particular assumption about the sea state is made, which allows to cope efficiently with any operating condition.

4) State and control constraints can be considered.

5) In addition to the wave excitation force, the state of the system can also be estimated simultaneously. These estimates can then be used for advanced control algorithms such as MPC.

The paper is organized as follows. In Section II, the mathematical model of the WEC considered in this work is briefly presented. Then the first estimation method, based on a Kalman filter, is proposed in Section III. Using a receding horizon filter, an alternative approach is presented in Section IV. In Section V, the experimental setup is described used to validate the algorithms is described, and the estimation results are reported. Some conclusions are drawn in Section VII.

\section{STATE-SPACE WEC MODELING}

We consider point-absorber WECs consisting of a heaving body that moves in one degree of freedom with respect to a fixed anchor or a submerged body (see Fig. 3). From the relative motion, useful energy can be extracted.

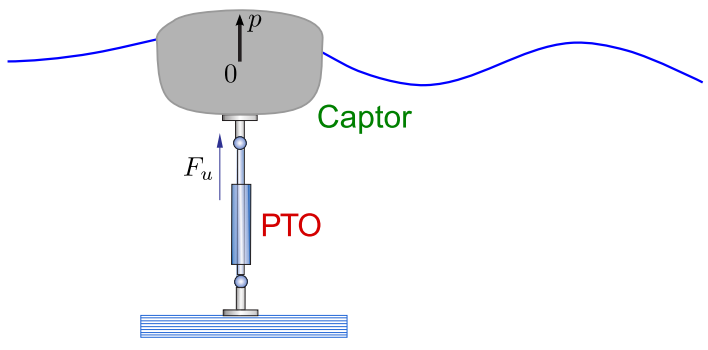

Fig. 3. One-degree-of-freedom heaving-body WEC.

Under the assumption that the oscillations of the system are relatively small and using Newton's second law, the WEC model can be expressed in the time domain as follows [4],

$$
M \ddot{p}(t)=F_{h d}(t)+F_{\text {rad }}(t)+F_{e x}(t)-F_{u}(t)
$$

where $p(t)$ is the heaving position with respect to the equilibrium point, $M$ is the WEC (captor) mass, $F_{h d}(t)$ is the hydrostatic force, $F_{\text {rad }}(t)$ is the radiation force, $F_{e x}(t)$ is the excitation force to be estimated, and $F_{u}(t)$ is the control force from the PTO system. Note that since the motion of the system is assumed to be relatively small, the drag force is considered negligible and hence not taken into account in the model (1).

It is assumed that $p(t)$ and $\dot{p}(t)$, the position and the velocity of the captor, as well as $F_{u}(t)$, the force applied by the PTO, can be measured or estimated. Note that in most literature on this subject, $F_{u}^{s p}(t)$, the output of the hydrodynamic controller, is used instead in the estimation algorithm. This corresponds to the implicit assumption that the low-level PTO force servo ensures perfect tracking of $F_{u}^{s p}(t)$. Indeed, if this assumption were justified by the characteristic of the WEC under study, one could replace $F_{u}(t)$ with $F_{u}^{s p}(t)$ in the following.

Remark: Newton's second law for linear motion is used to obtain a mathematical model of the WECs, because the motion is linear. There are other types of point-absorber WEC, where the (main) motion is angular. The quantity that fully 
describes the system in this case is the angular deviation from the equilibrium point. Newton's second law for rotation can be applied to obtain a mathematical model. The changes that take place are that the equivalent mass changes to an equivalent moment of inertia, and the acting forces become acting moments.

It is well known [4] that, for small $p(t)$, the hydrostatic force can be considered a linear function of $p(t)$, i.e.

$$
F_{h d}(t)=-K p(t)
$$

where $K$ is the hydrostatic stiffness coefficient. The radiation force is given as in [16]

$$
F_{\text {rad }}(t)=-M_{\infty} \ddot{p}(t)-F_{r}(t)
$$

where $M_{\infty}$ is the added mass at infinitely high frequency, and

$$
F_{r}(t)=\int_{0}^{t} h(t-\tau) \dot{p}(\tau) d \tau
$$

In (4), $h(t)$ is the impulse response function of the radiation force, which can be obtained by boundary element methods [17], [18], or approximated using analytical solutions for specific float geometry [19], [20].

To avoid performing the inconvenient convolution $F_{r}(t)=$ $\int_{0}^{t} h(t-\tau) \dot{p}(\tau) d \tau$, equation (4) can be considered as a linear system, where the input is $\dot{x}(t)$, and the output is $F_{r}(t)$. Using Prony's method [21] implemented in Matlab, the following state-space equation is obtained

$$
\begin{cases}\dot{r}(t) & =A_{r} r(t)+B_{r} \dot{p}(t) \\ F_{r}(t) & =C_{r} r(t)+D_{r} \dot{p}(t)\end{cases}
$$

where $\left(A_{r}, B_{r}, C_{r}, D_{r}\right)$ is a state-space realization, and $r(t)$ is an internal state with no particular physical meaning.

Combining (1), (2), (3), (5), one obtains

$$
\begin{cases}M \ddot{p}(t) & =-K p(t)-M_{\infty} \ddot{p}(t)-F_{r}(t)+F_{e x}(t)-F_{u}(t) \\ \dot{r}(t) & =A_{r} r(t)+B_{r} \dot{p}(t), \\ F_{r}(t) & =C_{r} r(t)+D_{r} \dot{p}(t)\end{cases}
$$

Define

$$
\left\{\begin{array}{l}
x_{1}(t)=p(t) \\
x_{2}(t)=\dot{p}(t)
\end{array}\right.
$$

Note that $x_{1}(t)$ and $x_{2}(t)$ are directly accessible, since $p(t)$, $\dot{p}(t)$ are measurable. Using (6), (7), one obtains

$$
\left\{\begin{aligned}
\dot{x}_{1}(t)= & x_{2}(t) \\
\dot{x}_{2}(t)= & -\frac{K}{M_{+}+M_{\infty}} x_{1}(t)-\frac{D_{r}}{M+M_{\infty}} x_{2}(t)- \\
& -\frac{C_{r}}{M+M_{\infty}} r(t)+\frac{1}{M+M_{\infty}}\left(F_{e x}(t)-F_{u}(t)\right) \\
\dot{r}(t)= & B_{r} x_{2}(t)+A_{r} r(t)
\end{aligned}\right.
$$

or equivalently

$$
\left\{\begin{array}{l}
\dot{x}(t)=A_{c} x(t)+B_{c} F_{e x}(t)-B_{c} F_{u}(t), \\
y(t)=C_{c} x(t)
\end{array}\right.
$$

where

$$
\left\{\begin{array}{l}
x(t)=\left[x_{1}(t) x_{2}(t) r^{T}(t)\right]^{T} \\
y(t)=\left[\begin{array}{l}
x_{1}(t) \\
x_{2}(t)
\end{array}\right]
\end{array}\right.
$$

and

$$
\begin{aligned}
A_{c} & =\left[\begin{array}{ccc}
0 & 1 & \mathbf{0} \\
-\frac{K}{M+M_{\infty}} & -\frac{D_{r}}{M_{r}} & -\frac{C_{r}}{M+M_{\infty}} \\
0 & B_{r} & A_{r}
\end{array}\right], \\
B_{c} & =\left[\begin{array}{c}
0 \\
\frac{1}{M+M_{\infty}} \\
\mathbf{0}
\end{array}\right], \quad C_{c}=\left[\begin{array}{lll}
1 & 0 & \mathbf{0} \\
0 & 1 & 0
\end{array}\right]
\end{aligned}
$$

where $\mathbf{0}$ is a zero matrix of appropriate dimension.

For the system (9), $F_{e x}(t)$ is not directly measurable. Our problem is to estimate $F_{e x}(t)$ using other measured quantities such as $y(t)$ and $F_{u}(t)$. For this purpose, (9) is discretized with the Tustin's method. As a result, the following system is obtained

$$
\begin{cases}x(k+1) & =A x(k)+B F_{e x}(k)-B F_{u}(k) \\ y(k) & =C x(k)+D F_{e x}(k)-D F_{u}(k)\end{cases}
$$

To simplify the description, denote

$$
w(k)=F_{e x}(k), u(k)=F_{u}(k)
$$

thus equation (10) is rewritten as

$$
\begin{cases}x(k+1) & =A x(k)+B w(k)-B u(k) \\ y(k) & =C x(k)+D w(k)-D u(k)\end{cases}
$$

\section{KALMAN FILTER APPROACH}

The main idea of the Kalman filter based approach is to consider the wave excitation force $w(k)$ as a state. As a consequence, a mathematical model is needed to relate $w(k)$ and $w(k+1)$. It should be noted that this is not a new idea, even for WECs. In [13], by assuming that $w(k)$ is the sum of a number of sinusoids with different phases and amplitudes, a mathematical model consisting of a bank of oscillators is built up. The number of sinusoids and their individual angular frequencies are fixed, and are tuning parameters. However, looking at the experimental estimation results reported in [13], it appears that

1) The amplitude of the wave excitation force is slightly overestimated.

2) There exists a significant signal delay in the estimated wave excitation force.

Note that the assumption on the constancy of the decomposed angular frequencies is rather strong, since it is well known that the spectrum of $w(k)$ is time-varying. In addition, in order to accurately describe $w(k)$, it is well known [17] that more than 10000 sinusoids are needed. Consequently, one might have a problem with the delay and/or with the computational complexity. Note also that the approach is not applicable if the wave excitation force has nonzero mean.

In this work, the following mathematical model is used

$$
w(k+1)=w(k)+\epsilon_{m}(k)
$$

where $\epsilon_{m}(k)$ describes the variation of $w(k)$ and is considered as a random number. The model (13) assumes that at each sampling time, the wave excitation force takes a random step away from its previous value, and the steps are independently and identically distributed in size. Clearly, the computational complexity is low, since (13) is simple. 
To take into account unmodeled dynamics and measurement noises, a more realistic model than (12) for the WEC system is introduced as

$$
\begin{cases}x(k+1) & =A x(k)+B w(k)-B u(k)+\epsilon_{x}(k) \\ y(k) & =C x(k)+D w(k)-D u(k)+\mu(k)\end{cases}
$$

where $\epsilon_{x}(k)$ describes unmodeled dynamics, and $\mu(k)$ describes measurement noises. Combining (13), (14), we obtain

$$
\begin{cases}x(k+1) & =A x(k)+B w(k)-B u(k)+\epsilon_{x}(k) \\ w(k+1) & =w(k)+\epsilon_{m}(k) \\ y(k) & =C x(k)+D w(k)-D u(k)+\mu(k)\end{cases}
$$

or equivalently

$$
\begin{cases}x_{a}(k+1) & =A_{a} x_{a}(k)+B_{a} u(k)+\epsilon(k) \\ y(k) & =C_{a} x(k)+D_{a} u(k)+\mu(k)\end{cases}
$$

where

$$
\begin{aligned}
& x_{a}(k)=\left[\begin{array}{c}
x(k) \\
w(k)
\end{array}\right], \epsilon(k)=\left[\begin{array}{c}
\epsilon_{x}(k) \\
\epsilon_{m}(k)
\end{array}\right], \\
& A_{a}=\left[\begin{array}{cc}
A & B \\
\mathbf{0} & 1
\end{array}\right], \quad B_{a}=\left[\begin{array}{c}
-B \\
0
\end{array}\right], \\
& C_{a}=\left[\begin{array}{ll}
C & D
\end{array}\right], D_{a}=-D
\end{aligned}
$$

Using (16), it follows that the problem of estimating $w(k)$ becomes a state estimation problem. One way to estimate the unknown state vector $x_{a}(k)$, that can take into account information about $\epsilon(k)$ and $\mu(k)$, is to apply the well-known linear Kalman filter (LKF) algorithm [22], [23].

The following assumptions are classical in the Kalman filter theory. $x_{a}(0)$ is a random vector that is uncorrelated to $\epsilon(k)$ and $\mu(k) . x_{a}(0)$ has a known mean $\bar{x}_{a}(0)$ with $P_{0}$ as a covariance matrix. $\epsilon(k)$ and $\mu(k)$ are uncorrelated, zeromean white-noise processes with covariance matrices $Q$ and $R$, respectively. The correlation technique in [24] is used to obtain $Q$ and $R$.

The following notations are used:

- $\hat{x}_{a}(k \mid k-1)$ is the estimate of $x_{a}(k)$ given measurements from time $k-1$.

- $\hat{x}_{a}(k \mid k)$ is the estimate of $x_{a}(k)$ given measurements from time $k$.

- $P(k \mid k-1)$ is the covariance matrix of $x_{a}(k)$ given measurements from time $k-1$.

- $P(k \mid k)$ is the covariance matrix of $x_{a}(k)$ given measurements from time $k$.

Then the LKF algorithm is summarized as follows

- Time-update equation

$\begin{cases}\hat{x}_{a}(k \mid k-1) & =A_{a} \hat{x}_{a}(k-1 \mid k-1)+B_{a} u(k-1), \\ P(k \mid k-1) & =A_{a} P(k-1 \mid k-1) A_{a}^{T}+Q\end{cases}$

- Measurement-update equation

$\begin{cases}K(k) & =P(k \mid k-1) C_{a}^{T}\left(C_{a} P(k \mid k-1) C_{a}^{T}+R\right)^{-1} \\ \hat{x}_{a}(k \mid k) & =\hat{x}_{a}(k \mid k-1)+ \\ & +K(k)\left(y(k)-C_{a} \hat{x}_{a}(k \mid k-1)-D_{a} u(k)\right) \\ P(k \mid k) & =\left(I-K(k) C_{a}\right) P(k \mid k-1)\end{cases}$

The Kalman filter based approach is summarized as follows.

Algorithm 1: Kalman filter - random-walk model approach
1) Inputs: $y(k)=\left[\begin{array}{ll}x_{1}(k) & x_{2}(k)\end{array}\right]^{T}$ (measurements of WEC system outputs), $u(k)$ (measurements of WEC system control input), $\hat{x}_{a}(k-1 \mid k-1), P(k-1 \mid k-1)$ (estimates at previous time instant), and the parameters $Q, R$ (covariance matrices)

2) Output: $\hat{w}(k)$ (estimate of wave excitation force)

3) Initialize: $k=0, \hat{x}_{a}(0 \mid 0)=\bar{x}_{a}(0), P(0 \mid 0)=P_{0}$

4) At each time instant

(i) Apply the linear Kalman filter to obtain $\hat{x}_{a}(k \mid k)$, $P(k \mid k)$

(ii) Compute the estimate of wave excitation force $\hat{w}(k)$ as $\hat{w}(k)=\left[\begin{array}{ll}\mathbf{0} & 1\end{array}\right] \hat{x}_{a}(k \mid k)$

Let us stress that, since the assumption on the random-walk model of $w(k)$ is quite general, the approach can be applied to almost any kind of wave excitation force signals.

\section{RECEDING HORIZON ESTIMATION APPROACH}

In the previous section, by imposing a random-walk model dynamics for the wave excitation force, the state of the system is first augmented. Then the classical linear Kalman filter is used to obtain an estimate of the augmented state. However, the assumption (13) on the behavior of $w(k)$ makes the approach sub-optimal.

Using a receding-horizon principle, a new estimation algorithm is proposed in this section. The main advantages of the approach are:

- There is no assumption on the dynamics of the wave excitation force.

- Information about process and measurement noises can be considered.

- Input and state constraints can be naturally included.

Let us start again from a WEC model, that considers unmodeled dynamics and measurement noises

$$
\begin{cases}x(k+1) & =A x(k)+B w(k)-B u(k)+\epsilon(k), \\ y(k) & =C x(k)+D w(k)-D u(k)+\mu(k)\end{cases}
$$

where $\epsilon(k)$ and $\mu(k)$ are respectively, the process and the measurement noises. It is assumed that $\epsilon(k)$ and $\mu(k)$ are white noises with zero mean.

Receding-horizon estimation is an algorithm, that recursively estimates the state and the unknown input by considering a finite window of data. The problem consists in estimating, at any time instant $k \geq N$, the state vector $x(k)$ and the unknown input $M_{e x}(k)$, on the basis of

- A priori estimators: $\hat{x}(k-N \mid k-1), \hat{w}(k-N \mid k-1)$.

- Output information: $y(k-N), y(k-N+1), \ldots, y(k)$.

- Input information: $u(k-N), u(k-N+1), \ldots, u(k)$.

Here $\hat{x}(k-N \mid k-1)$ and $\hat{w}(k-N \mid k-1)$ denote the estimates of $x(k-N)$ and $w(k-N)$ given the measurements from time $k-1$.

With a slight abuse of notation, let us denote $\hat{x}_{0}=\hat{x}(k-$ $N \mid k-1)$ and $\hat{w}_{0}=\hat{w}(k-N \mid k-1)$. Let us denote also $x_{j}=$ $\hat{x}(k-N+j \mid k), w_{j}=\hat{w}(k-N+j \mid k)$, and $\epsilon_{j}=\hat{\epsilon}(k-N+j \mid k)$, for $j=0,1, \ldots N$. The moving horizon problem is formulated as, at each time instant $k \geq N$

$$
\min _{x_{0}, w_{0}, w_{1}, \ldots, w_{N}, \epsilon_{1}, \ldots, \epsilon_{N}} J\left(x_{0}, w_{0}, w_{1}, \ldots, w_{N}, \epsilon_{1}, \ldots, \epsilon_{N}\right)
$$


where the cost function is defined as

$$
\begin{aligned}
& J(\cdot)=\left\|x_{0}-\hat{x}_{0}\right\|_{P_{0}^{-1}}^{2}+\left\|w_{0}-\hat{w}_{0}\right\|_{Q_{0}^{-1}}^{2}+\sum_{j=0}^{N-1}\left\|\epsilon_{j}\right\|_{Q^{-1}}^{2}+ \\
& +\sum_{j=1}^{N}\left\|y(k-N+j)-C x_{j}\right\|_{R^{-1}}^{2}+\lambda \sum_{j=1}^{N}\left\|w_{j}-w_{j-1}\right\|^{2}
\end{aligned}
$$

Here $\|x\|_{M}$ denotes $x^{T} M x$ for a real vector $x$ and a real symmetric matrix $M$. There are five terms in (19):

- The sum of the first term $\left\|x_{0}-\hat{x}_{0}\right\|_{P_{0}^{-1}}^{2}$ and the second term $\left\|w_{0}-\hat{w}_{0}\right\|_{Q_{0}^{-1}}^{2}$ is called the arrival cost. It summarizes the prior information at time $k-N$ and is part of the data of the estimation problem.

- The third and fourth terms penalize the process noise and the measurement noise via the weighting matrices $Q$, and $R$, respectively.

- The last term is a regularization term. It exploits the fact that the wave excitation force is a smooth signal. To the best of the authors' knowledge, this is the first time, not only in WEC literature, but also in moving horizon estimation literature, that such a term is considered.

Remark: Another way of using information about the wave excitation force smoothness is to impose the rate constraints $\left|w_{j}-w_{j-1}\right| \leq S, \quad j=1,2, \ldots, N$, where $S>0$ is the maximal variation of $w_{j}$.

Next we will transform (18), (19) into a quadratic programming problem. By propagating (17) in time, one gets

$$
\hat{Y}=\Phi_{y} x_{0}+\Psi_{y} W-\Psi_{y} U+\Gamma_{y} E
$$

where

$$
\begin{aligned}
& \hat{Y}=\left[\begin{array}{ccccc}
\hat{y}(k-N+1)^{T} & \hat{y}(k-N+2)^{T} & \ldots & \hat{y}(k)^{T}
\end{array}\right]^{T}, \\
& \Psi_{y}=\left[\begin{array}{ccccc}
C B & D & \ldots & 0 & 0 \\
C A B & C B & \ldots & 0 & 0 \\
\vdots & \vdots & \ddots & \vdots & \vdots \\
C A^{N-1} B & C A^{N-2} B & \ldots & C B & D
\end{array}\right], \\
& \Phi_{y}=\left[\begin{array}{c}
C A \\
C A^{2} \\
\vdots \\
C A^{N}
\end{array}\right], \Gamma_{y}=\left[\begin{array}{cccc}
C A & C & \ldots & 0 \\
\vdots & \vdots & \ddots & \vdots \\
C A^{N-1} & C A^{N-2} & \ldots & C
\end{array}\right]
\end{aligned}
$$

Rewrite the cost function (19) as

$$
\begin{aligned}
J= & \left\|x_{0}-\hat{x}_{0}\right\|_{P_{0}^{-1}}^{2}+\left\|w_{0}-\hat{w}_{0}\right\|_{Q_{0}^{-1}}^{2}+ \\
& +E^{T} \mathbf{Q} E+(Y-\hat{Y})^{T} \mathbf{R}(Y-\hat{Y})+W^{T} \Lambda W
\end{aligned}
$$

where $\mathbf{Q}=\operatorname{diag}\left(Q^{-1}\right), \mathbf{R}=\operatorname{diag}\left(R^{-1}\right)$, and

$$
\Lambda=\lambda\left[\begin{array}{ccccccc}
y(k-N+1)^{T} & y(k-N+2)^{T} & \ldots & y(k)^{T}
\end{array}\right]^{T},
$$

$\operatorname{diag}(X)$ denotes a square diagonal matrix, whose diagonal elements are $X$. Substituting (20) into (21), one gets

$$
\begin{aligned}
J=\left\|x_{0}-\hat{x}_{0}\right\|_{P^{-1}}^{2}+\left\|w_{0}-\hat{w}_{0}\right\|_{Q_{0}^{-1}}^{2}+ \\
+E^{T} \mathbf{Q} E+\mathcal{G}^{T} \mathbf{R} \mathcal{G}+W^{T} \Lambda W
\end{aligned}
$$

with $\mathcal{G}=Y-\Phi_{y} x_{0}-\Psi_{y} W+\Psi_{y} U-\Gamma_{y} E$. Or, equivalently

$$
\begin{aligned}
& J=x_{0}^{T} P_{0}^{-1} x_{0}-2 \hat{x}_{0}^{T} P_{0}^{-1} x_{0}+\hat{x}_{0}^{T} P_{0}^{-1} \hat{x}_{0}+w_{0}^{T} Q_{0}^{-1} w_{0}- \\
& -2 \hat{w}_{0}^{T} Q_{0}^{-1} w_{0}+\hat{w}_{0}^{T} Q_{0}^{-1} \hat{w}_{0}+E^{T} \mathbf{Q} E+\mathcal{G}^{T} \mathbf{R} \mathcal{G}+W^{T} \Lambda W
\end{aligned}
$$

The terms $\hat{x}_{0}^{T} P_{0}^{-1} \hat{x}_{0}$ and $\hat{w}_{0}^{T} Q_{0}^{-1} \hat{w}_{0}$ can be removed, since they do not influence the optimal argument. As the result

$$
\begin{aligned}
J_{1}= & x_{0}^{T} P_{0}^{-1} x_{0}-2 \hat{x}_{0}^{T} P_{0}^{-1} x_{0}+w_{0}^{T} Q_{0}^{-1} w_{0}- \\
& -2 \hat{w}_{0}^{T} Q_{0}^{-1} w_{0}+E^{T} \mathbf{Q} E+\mathcal{G}^{T} \mathbf{R} \mathcal{G}+W^{T} \Lambda W
\end{aligned}
$$

Define

$$
\begin{aligned}
& \left\{\begin{array}{l}
\xi=\left[\begin{array}{lll}
W^{T} & x_{0}^{T} & E^{T}
\end{array}\right]^{T} \\
V=Y+\Psi_{y} U \\
\Psi_{s}=\left[\begin{array}{lll}
\Psi_{y} & \Phi_{y} & \Gamma_{y}
\end{array}\right]
\end{array}\right. \\
& \begin{aligned}
\Lambda_{1} & =\left[\begin{array}{ccccccc}
Q_{0}^{-1}-\lambda & \lambda & 0 & 0 & \ldots & 0 & 0 \\
0 & -\lambda & \lambda & 0 & \ldots & 0 & 0 \\
0 & 0 & -\lambda & \lambda & \ldots & 0 & 0 \\
\vdots & \vdots & \vdots & \vdots & \ddots & \vdots & \vdots \\
0 & 0 & 0 & 0 & \ldots & \lambda & 0 \\
0 & 0 & 0 & 0 & \ldots & -\lambda & \lambda
\end{array}\right], \\
\mathbf{P} & =\left[\begin{array}{ccc}
\Lambda_{1} & 0 & 0 \\
0 & P_{0}^{-1} & 0 \\
0 & 0 & \mathbf{Q}
\end{array}\right], \mathbf{S}=\left[\begin{array}{ccccc}
Q_{0}^{-1} & 0 & \ldots & 0 \\
0 & P_{0}^{-1} & \ldots & 0
\end{array}\right]
\end{aligned}
\end{aligned}
$$

The cost (23) can be rewritten as

$$
J_{1}=\xi^{T} \mathbf{P} \xi-2\left[\begin{array}{ll}
\hat{w}_{0}^{T} & \hat{x}_{0}^{T}
\end{array}\right] \mathbf{S} \xi+\left(V-\Psi_{s} \xi\right)^{T} \mathbf{R}\left(V-\Psi_{s} \xi\right)
$$

or equivalently

$$
\begin{aligned}
& J_{1}=\xi^{T} \mathbf{P} \xi-2\left[\begin{array}{ll}
\hat{w}_{0}^{T} & \hat{x}_{0}^{T}
\end{array}\right] \mathbf{S} \xi+\xi^{T} \Psi_{s}^{T} \mathbf{R} \Psi_{s} \xi- \\
& -2 V^{T} \mathbf{R} \Psi_{s} \xi+V^{T} \mathbf{R} V
\end{aligned}
$$

The term $V^{T} \mathbf{R} V$ can be omitted in the cost function (25) since it does not influence the optimal argument. As a result

$$
J_{2}=\xi^{T}\left(\mathbf{P}+\Psi_{s}^{T} \mathbf{R} \Psi_{s}\right) \xi-2\left[\begin{array}{lll}
\hat{w}_{0}^{T} & \hat{x}_{0}^{T} & V^{T}
\end{array}\right]\left[\begin{array}{c}
\mathbf{S} \\
\mathbf{R} \Psi_{s}
\end{array}\right] \xi
$$

It is worth noticing that the bounds on the wave excitation force and the state can be considered in the moving-horizon estimation framework by including the following constraints

$$
\left\{\begin{array}{l}
w \in P_{w}, \quad P_{w}=\left\{w: H_{w} w \leq g_{w}\right\} \\
x \in P_{x}, \quad P_{x}=\left\{x: H_{x} x \leq g_{x}\right\}
\end{array}\right.
$$

where $g_{w}>0$ and $g_{x}>0$. This condition implies that the origin is contained in the interior of $P_{w}$ and $P_{x}$.

Using (24), the constraints (27) can be expressed as a linear inequality

$$
H_{\xi} \xi \leq g_{\xi}+H_{d} U
$$

Combining (26), (28), the moving horizon estimation problem boils down to

$$
\begin{aligned}
& \min _{\xi}\left\{\xi^{T}\left(\mathbf{P}+\Psi_{s}^{T} \mathbf{R} \Psi_{s}\right) \xi-2\left[\begin{array}{lll}
\hat{w}_{0}^{T} & \hat{x}_{0}^{T} & V^{T}
\end{array}\right]\left[\begin{array}{c}
\mathbf{S} \\
\mathbf{R} \Psi_{s}
\end{array}\right] \xi\right\} \\
& \text { s.t. } H_{\xi} \xi \leq g_{\xi}+H_{d} U
\end{aligned}
$$

This is a constrained quadratic programming problem, for which nowadays, there exist several effective solvers, e.g. [25]. 
Let $\xi^{*}$ be the optimal solution of (29), the estimate of the wave excitation forces is, from given measurement at time $k$

$$
\hat{w}(k-N+j \mid k)=\xi^{*}(j), \quad \forall j=1,2, \ldots, N
$$

Remark: When the constraints are not active, there exists a unique analytical solution to (29)

$$
\xi^{*}=\left(\mathbf{P}+\Psi_{s}^{T} \mathbf{R} \Psi_{s}\right)^{-1}\left[\begin{array}{ll}
\mathbf{S}^{T} & \Psi_{s}^{T} \mathbf{R}^{T}
\end{array}\right]\left[\begin{array}{c}
\hat{w}_{0} \\
\hat{x}_{0} \\
V
\end{array}\right]
$$

The moving horizon estimation algorithm is summarized as follows

Algorithm 2: Moving horizon estimation - unknown input approach

1) Inputs: $y(k)=\left[\begin{array}{ll}x_{1}(k) & x_{2}(k)\end{array}\right]^{T}$ (measurements of WEC system outputs), control input $u(k)$ (measurement of WEC system control output), $\hat{x}(k-N \mid k-1)$, $\hat{w}(k-N \mid k-1)$ (estimates from previous time instant)

2) Output: $\hat{w}(k \mid k)$

3) At each time instant $k$

(i) If $k \geq N$ then go to Step (ii). Wait, otherwise.

(ii) Solve the QP problem (29) to obtain $\xi^{*}$.

(iii) The estimate of the wave excitation force $w(k)$ can be calculated as $\hat{w}(k \mid k)=\xi^{*}(N)$.

\section{EXPERIMENTAL RESULTS}

\section{A. Experimental setup}

A laboratory prototype of a point absorber WEC [26] on a 1:20 scale with respect to the well-known Wavestar device installed near Hanstholm in Denmark from 2009 to 2013, was used to test the wave excitation force estimation methods described in the previous section. The tests were carried out within a broader campaign to assess the performance of a full MPC control system [27], as depicted in Fig. 2. As it can be seen in Fig. 4, the prototype consists of a float attached to an arm, which in turn is attached to a PTO emulator. The laboratory model is equipped with a laser and an acceleration sensor to measure, respectively, the position and the acceleration. The velocity of the float can then be estimated from these measurements using a linear Kalman filter.
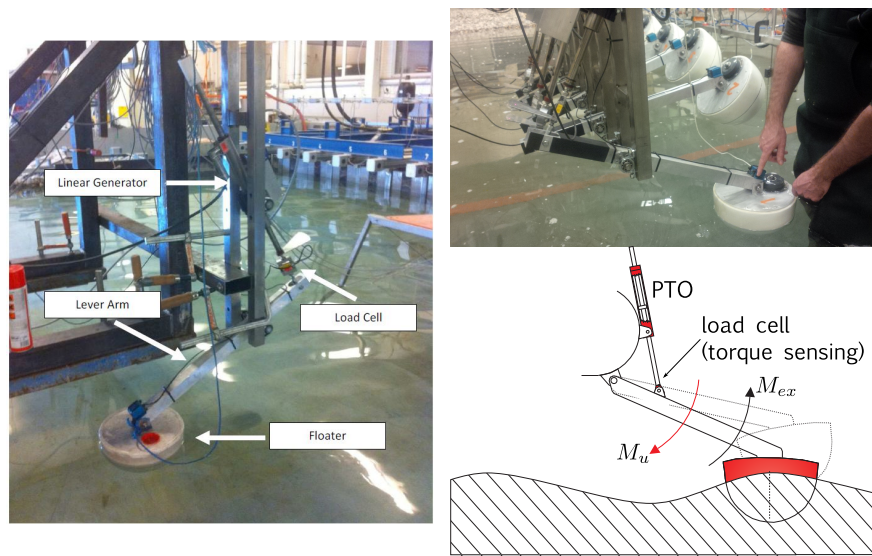

Fig. 4. Experimental set-up with laboratory model of the Wavestar device.
A linear electrical generator is chosen to emulate the PTO system. It applies a force to the arm, which can be measured by a load cell, see Fig. 4 and used to compute the equivalent moment applied to the float. The WEC controller sends a force reference to a low-level force servo-controller that operates the generator.

Note that for this application, we estimate a wave excitation moment instead of a wave excitation force, since the motion is rotational.

\section{B. Available data}

The data were collected in a wave basin of Aalborg University. The basin has a length of $15 \mathrm{~m}$, a width of $8 \mathrm{~m}$ and a maximum water depth of $0.7 \mathrm{~m}$. The wave paddles are driven by a total of 15 hydraulic pistons moving in the horizontal direction. The waves were generated by the wave maker based on the Pierson-Moskowitz spectrum [28]. Three distinguished features of the system were used thoroughly for testing. First the paddle movement can be stored during a test run and then can be reproduced in further test runs. Secondly, a trigger signal is sent by the wave generator, allowing exact alignment of the tests. Thirdly, the force sensor of the PTO can be used to measure the wave excitation moment, with the float being manually blocked at equilibrium position when the wave passes by.

These three features allow to compare the measured wave excitation moment as defined by linear wave theory from a first reference test to the estimated wave excitation moment from the following tests, where the float is moving and the PTO is turned on.

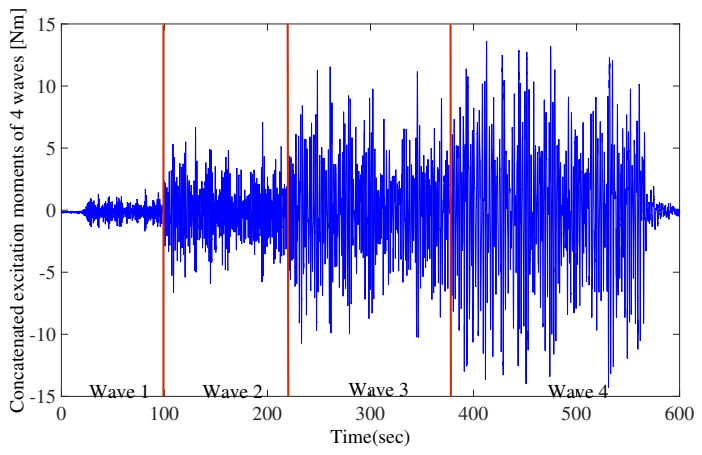

Fig. 5. Concatenation of four irregular waves (wave moment in $\mathrm{Nm}$ ).

For the present analysis, a concatenation of four different irregular waves representative of real-life sea conditions is considered, see Fig. 5. It can be observed that the waves were generated, by amplitude, from the smallest to the largest ones. The waves are unidirectional, two dimensional, longcrested waves. The four data sets were measured at a sampling frequency of $1 \mathrm{kHz}$, and down-sampled with a factor of 10 to reduce computational complexity. The spectra of the excitation waves 3 and 4 is presented in Fig. 6.

\section{Estimation results}

Fig. 7 presents the estimation results of the two algorithms applied to wave 3 . It can be observed that the results are 


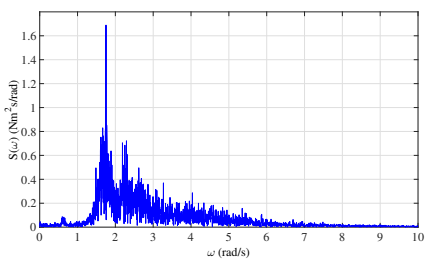

(a)

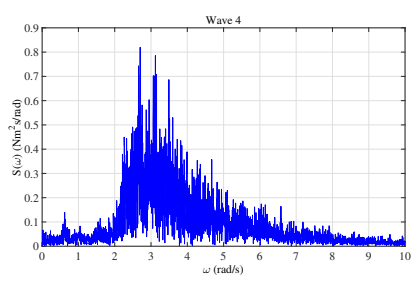

(b)
Fig. 6. Spectra of wave 3 and wave 4 .

good with both algorithms, though the estimates obtained with Algorithm 2 are smoother. In order to show the ability of our approaches to deal with the sea state time-varying nature, Fig. 8 compares the real wave moment to its estimates computed by the two algorithms for the transitory period from wave 3 to wave 4 .

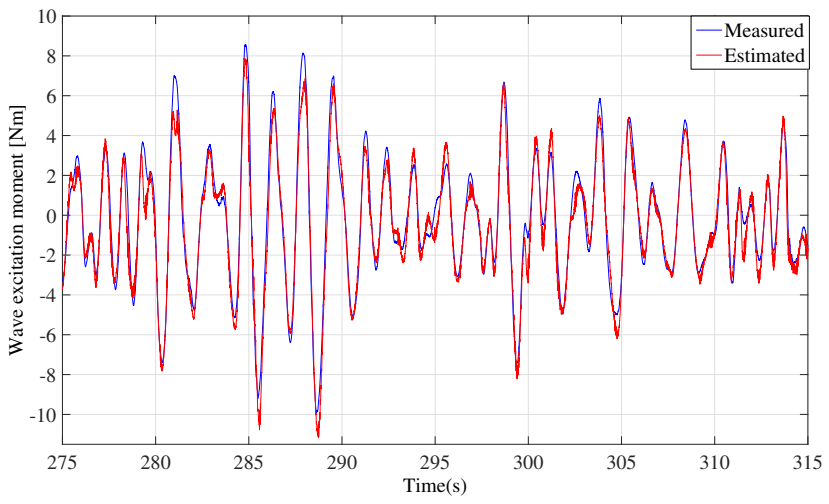

(a) Algorithm 1

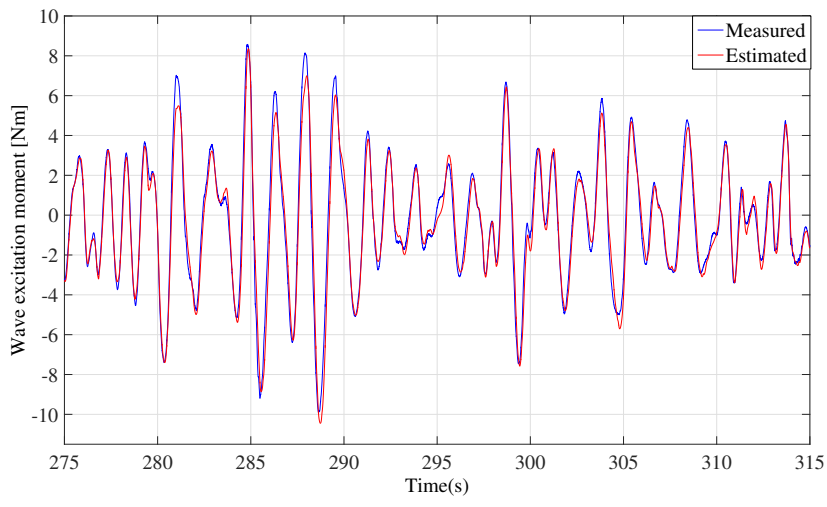

(b) Algorithm 2

Fig. 7. Measured and estimated wave excitation moments for wave 3 .

For advanced WEC control methods, it is strongly desirable to have an wave excitation moment estimation without any significant time delay. In this paper, the cross-correlation analysis technique is used to compute the maximum delay of the estimated wave excitation moment with respect to the reference real wave. The results are presented in Table I. A positive delay in Table I, means that the obtained estimated signal is advanced with respect to the reference signal. In the other case, the delay is negative. It should be noted that all the delays are less than 0.05, the sampling period chosen for MPC. It can also be observed that Algorithm 1 produces averagely

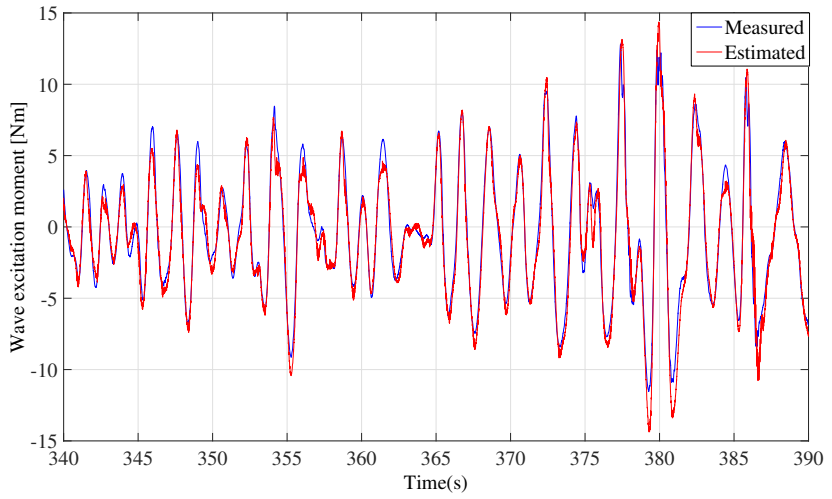

(a) Algorithm 1

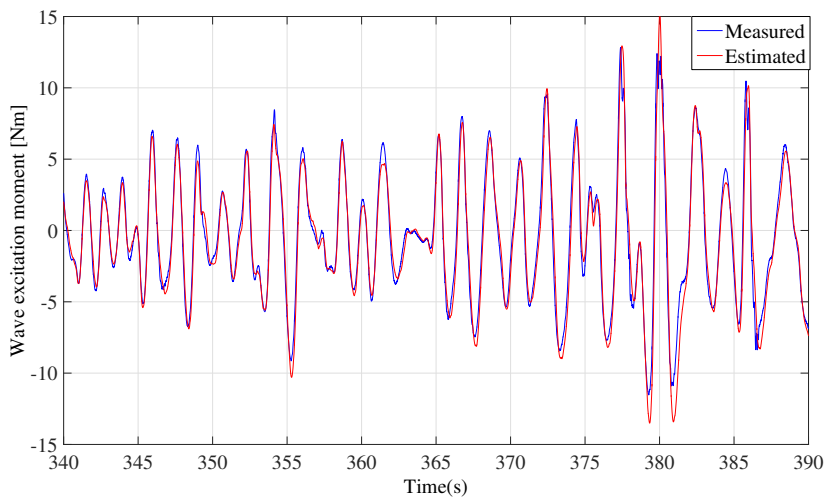

(b) Algorithm 2

Fig. 8. Measured and estimated wave excitation moments for the transitory period from wave 3 to wave 4 .

the best estimates with respect to the minimum (absolute) time-delay criterion. Algorithm 2, however, seems to have an anticipating effect, which may prove interesting for model predictive control.

\begin{tabular}{|c|c|c|c|c|}
\hline & Wave 1 & Wave 2 & Wave 3 & Wave 4 \\
\hline Algorithm 1 & $0.040 \mathrm{~s}$ & $0.010 \mathrm{~s}$ & $-0.005 \mathrm{~s}$ & $-0.020 \mathrm{~s}$ \\
\hline Algorithm 2 & $-0.005 \mathrm{~s}$ & $-0.025 \mathrm{~s}$ & $-0.035 \mathrm{~s}$ & $-0.050 \mathrm{~s}$ \\
\hline
\end{tabular}

TABLE I

TIME-DELAY ANALYSIS FOR DIFFERENT ALGORITHMS AND WAVES.

Finally, Table II presents a goodness-of-fit (GoF) index computed using the normalized mean square error (NMSE) criterion, as follows:

$$
m=\left(1-\sum_{k=0}^{N}\left(w_{e}(k)-w(k)\right)^{2} / \sum_{k=0}^{N} w^{2}(k)\right) 100 \%
$$

where $N$ is the number of data, $w_{e}(k)$ is the estimated wave force, and $w(k)$ is the reference wave force.

\begin{tabular}{|c|c|c|c|c|}
\hline & Wave 1 & Wave 2 & Wave 3 & Wave 4 \\
\hline Algorithm 1 & $86.05 \%$ & $92.36 \%$ & $94.58 \%$ & $94.62 \%$ \\
\hline Algorithm 2 & $91.93 \%$ & $94.64 \%$ & $94.90 \%$ & $94.08 \%$ \\
\hline
\end{tabular}

TABLE II

NMSE GOODNESS-OF-FIT ANALYSIS FOR DIFFERENT ALGORITHMS AND DIFFERENT WAVES.

Table II shows that the estimation algorithms give better result with higher and stronger waves. Algorithm 2 produces 
the best estimation result with respect to the maximum of the GoF criterion. However, this algorithm is computationally more demanding as it requires the solution of a quadratic programming problem at each time instant.

\section{ACKNOWLEDMENTS}

The authors wish to thank Aalborg University, and in particular Prof. Morten Kramer, for providing access to the experimental facility, as well as data and precious support.

\section{CONCLUSION}

One major difficulty in applying advanced control algorithms for wave energy converter systems resides in the fact that the wave excitation force is unmeasurable in real time. In this paper, two novel estimation methods are developed using position, velocity and PTO force measurements (or estimates). The first approach considers the wave excitation force as a system state, and applies a linear Kalman filter. A simple random-walk model is used to describe the dynamics of wave excitation force, which gives the algorithm the ability to deal with a broad range of varying sea-states with no need of recalibrating its parameters. The second approach views the wave excitation force as an unknown input. A receding horizon estimation algorithm, that can take into account the state and input constraints, is presented. An original feature of the algorithm is the regularization term introduced in the cost function, to improve the accuracy of the estimates.

The proposed estimation methods are evaluated by using real measurements, from an experimental set-up built around a laboratory-scale model of the well-known Wavestar pointabsorber. Both algorithms produce good estimation results, with a negligible time delay between the estimated and the real wave excitation force. Estimates obtained with the second algorithm are smoother and slightly more accurate, but at the expense of an increased computational complexity. Both estimation methods can be advantageously combined with advanced control algorithms to improve energy capture of WECs. They could also be used to monitor WEC operating conditions in real-time.

Further research is needed to verify if this approach could be applied to WECs with more complex dynamics. The inclusion of nonlinear terms such as drag forces should be addressed as well as the extension to multiple degree-of-freedom motion.

\section{REFERENCES}

[1] U. A. Korde and J. Ringwood, Hydrodynamic Control of Wave Energy Devices. Cambridge University Press, 2016.

[2] S. Salter, "The development of the duck concept," in Proc. Wave Energy Conference, 1978, pp. 17-27.

[3] E. V. Sánchez, R. H. Hansen, and M. M. Kramer, "Control performance assessment and design of optimal control to harvest ocean energy," IEEE Journal of Oceanic Engineering, vol. 40, no. 1, pp. 15-26, 2015.

[4] J. Falnes, Ocean waves and oscillating systems: linear interactions including wave-energy extraction. Cambridge university press, 2002.

[5] K. Budal and J. Falnes, "Wave power conversion by point absorbers: a norwegian project," International Journal of Ambient Energy, vol. 3, no. 2 , pp. 59-67, 1982.

[6] A. Babarit and A. Clément, "Optimal latching control of a wave energy device in regular and irregular waves," Applied Ocean Research, vol. 28, no. 2, pp. 77-91, 2006.
[7] A. Babarit, G. Duclos, and A. Clément, "Comparison of latching control strategies for a heaving wave energy device in random sea," Applied Ocean Research, vol. 26, no. 5, pp. 227-238, 2004.

[8] F. Saupe, Y. Creff, J.-C. Gilloteaux, P. Bozonnet, and P. Tona, "Latching control strategies for a heaving buoy wave energy generator in a random sea," in 19th IFAC World Congress, vol. 19, no. 1, 2014, pp. 7710-7716.

[9] J. Hals, J. Falnes, and T. Moan, "Constrained optimal control of a heaving buoy wave-energy converter," Journal of Offshore Mechanics and Arctic Engineering, vol. 133, no. 1, p. 011401, 2011.

[10] J. A. Cretel, G. Lightbody, G. P. Thomas, and A. W. Lewis, "Maximisation of energy capture by a wave-energy point absorber using model predictive control," in Proceedings of the 18th IFAC World Congress, Milano, Italy, Aug, 2011, pp. 3714-3721.

[11] G. Li and M. R. Belmont, "Model predictive control of sea wave energy converters-part i: A convex approach for the case of a single device," Renewable Energy, vol. 69, pp. 453-463, 2014.

[12] F. Fusco and J. V. Ringwood, "A simple and effective real-time controller for wave energy converters," Sustainable Energy, IEEE Transactions on, vol. 4, no. 1, pp. 21-30, 2013.

[13] P. Kracht, S. Perez-Becker, J.-B. Richard, and B. Fischer, "Performance improvement of a point absorber wave energy converter by application of an observer-based control: Results from wave tank testing," IEEE Transactions on Industry Applications, vol. 51, no. 4, pp. 3426-3434, 2015.

[14] B. A. Ling and B. A. Batten, "Real time estimation and prediction of wave excitation forces on a heaving body," in ASME 2015 34th international conference on ocean, offshore and arctic engineering, 2015.

[15] O. Abdelkhalik, S. Zou, R. Robinett, G. Bacelli, and D. Wilson, "Estimation of excitation forces for wave energy converters control using pressure measurements," International Journal of Control, pp. 1-13, 2016.

[16] W. Cummins, "The impulse response function and ship motions," DTIC Document, Tech. Rep., 1962.

[17] J. N. Newman, Marine hydrodynamics. MIT press, 1977.

[18] C.-H. Lee and J. N. Newman, "Wamit user manual," WAMIT, Inc, 2006.

[19] T. Havelock, "Waves due to a floating sphere making periodic heaving oscillations," in Proceedings of the Royal Society of London A: Mathematical, Physical and Engineering Sciences, vol. 231, no. 1184. The Royal Society, 1955, pp. 1-7.

[20] A. Hulme, "The wave forces acting on a floating hemisphere undergoing forced periodic oscillations," Journal of Fluid Mechanics, vol. 121, pp. 443-463, 1982

[21] G. De Backer, "Hydrodynamic design optimization of wave energy converters consisting of heaving point absorbers," Ph.D. dissertation, Gent University, 2009.

[22] R. E. Kalman and R. S. Bucy, "New results in linear filtering and prediction theory," Journal of Fluids Engineering, vol. 83, no. 1, pp. 95-108, 1961

[23] M. S. Grewal and A. P. Andrews, Kalman filtering: Theory and Practice with MATLAB. John Wiley \& Sons, 2014.

[24] R. Mehra, "On the identification of variances and adaptive Kalman filtering," IEEE Transactions on automatic control, vol. 15, no. 2, pp. $175-184,1970$.

[25] J. Mattingley and S. Boyd, "CVXGEN: a code generator for embedded convex optimization," Optimization and Engineering, vol. 13, no. 1, pp. $1-27,2012$.

[26] A. S. Zurkinden, M. Kramer, M. T. Teimouri, and M. Alves, "Comparison between numerical modeling and experimental testing of a point absorber wec using linear power take-off system," in ASME International Conference on Offshore Mechanics and Arctic Engineering, 2012.

[27] H.-N. Nguyen, G. Sabiron, P. Tona, M. Kramer, and E. Vidal-Sanchez, "Experimental validation of a nonlinear MPC strategy for a wave energy converter prototype," in ASME International Conference on Offshore Mechanics and Arctic Engineering, 2016.

[28] W. J. Pierson and L. Moskowitz, "A proposed spectral form for fully developed wind seas based on the similarity theory of S.A. Kitaigorodskii," Journal of geophysical research, vol. 69, no. 24, pp. 5181-5190, 1964. 